Bulk and Surface Electronic Structure of Actinide, Rare Earth, and Transition Metal Elements and Compounds

Author(s):

John W. Wills, T-1

PECEMPD

JUL 131903

Olle Eriksson, Uppsala University

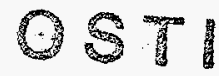

Submitted to:

DOE Office of Scientific and Technical Information (OSTI)

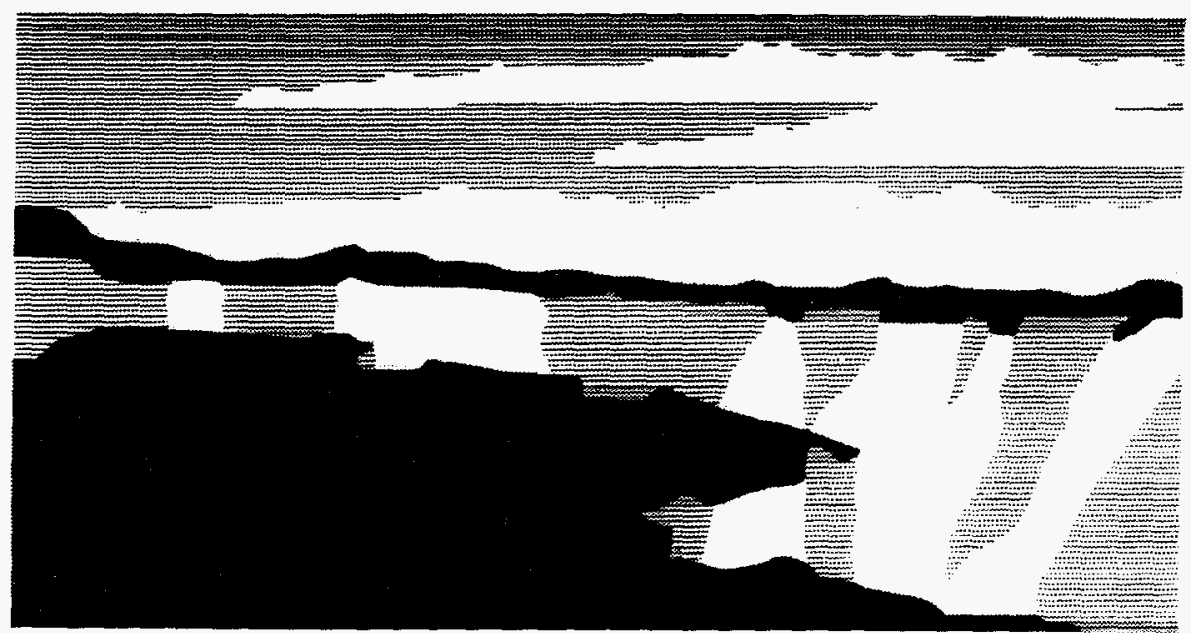

LOS Alamos National Laboratory, an affirmative action/equal opportunity employer, is operated by the University of California for the U.S. Department of Energy under contract W-7405-ENG-36. By acceptance of this article, the publisher recognizes that the U.S. Government retains a nonexclusive, royaltyfree license to publish or reproduce the published form of this contribution, or to allow others to do so, for U.S. Govemment purposes. The Las Alamos

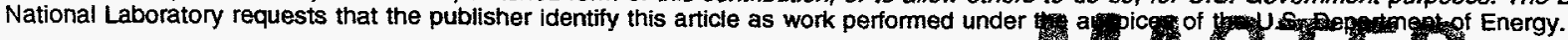




\section{DISCLAIMER}

Portions of this document may be illegible in electronic image products. Images are produced from the best available original document. 


\title{
Bulk and Surface Electronic Structure of Actinide, Rare Earth, and Transition Metal Elements and Compounds
}

\author{
John M. Wills* and Olle Eriksson (Uppsala University, Sweden)
}

\begin{abstract}
This is the final report for a three-year, Laboratory Directed Research and Development (LDRD) project at the Los Alamos National Laboratory (LANL). This project sought to study of unusual magnetic and structural properties of rare earth, actinide, and transition metals through high-precision electronic structure calculations. Magnetic moment anisotropies in bulk and surface systems were studied, with emphasis on novel surfaces with unusual magnetic properties with possible applicability in magnetic recording. The structural stability, bonding properties, and elastic response of the actinides, as well as transition and rare earth elements and compounds, were also studied. The project sought to understand the unusual crystallographic and cohesive properties of the actinides and the importance of correlation to structural stability and the nature of the delocalization transition in these elements. Theoretical photoemission spectra, including surface effects, were calculated for rare earths and actinides.
\end{abstract}

\section{Background and Research Objectives}

This project has supported a theoretical study, by means of high precision calculations, of the electronic-structure related properties of transition metal, actinide, and rare earth elements and compounds. The principal tool for this study has been a total energy, all electron, electronic structure method, developed at LANL for both bulk and surface systems, capable of an accuracy equaling or surpassing that of other existing techniques. The successes of this method prior to this work have included the first successful theoretical phase diagrams for $\mathrm{Ce}$ and the light actinides [1]. The study was focused on the magnetic and structural properties of transition, rare earth, and actinide elements and compounds.

\footnotetext{
* Principal investigator, e-mail: jxw@lanl.gov
} 
During the last decades it has been shown that bulk magnetic moments (spin and orbital) can be calculated with fair accuracy. More interesting aspects of magnetic behavior, however, have eluded theoretical description because calculational techniques have not had the required precision. For example, very few successful attempts at calculating magnetic anisotropies have been published. Furthermore, most previous theoretical work has not been able to deal successfully with surface magnetism, which is unfortunate since two-dimensional magnetic systems can be quite different from the bulk systems with new and exotic phenomena occurring. We have, for example, predicted that $\mathrm{Ru}$ and $\mathrm{Rh}$ as monolayers on $\mathrm{Ag}$ are magnetic [2], a behavior not observed in bulk Ru and $\mathrm{Rh}$. Recent experiments on surfaces of alpha-Ce and $\mathrm{Ce}$ surfaces on $\mathrm{W}$ show new and interesting phenomena, indicating spin polarization at the surface. Since relativistic effects are known to be important, $\mathrm{Ce}$ and the light actinides are ideal systems for studying surface anisotropy, with the possibility of large magneto-optic Kerr angles. The goal of this part of the research has been to study magnetic anisotropy and enhanced magnetic moments in bulk and surface transition metals and actinides.

A rudimentary understanding of the structural and bonding properties of the elemental transition metals and actinides has been gained from ab-initio electronic structure calculations in the local density approximation (LDA), presenting a picture of 5f-bonding in the early actinides similar to d-bonding in the transition metals, interrupted by $5 \mathrm{f}$ localization at Am. With the advent of high speed computers and the development of sophisticated electronic structure techniques, the structural and elastic properties of complex solids have become accessible to $a b$-initio calculation. This part of this project has been directed toward the study of structural stability and elastic response of actinide and transition elements and compounds. An important part of this research is the study of the differences in structural properties between transition metals and actinides such as the complicated crystal structures found in the early actinides, the increase in equilibrium volume going from $\mathrm{Np}$ to $\mathrm{Pu}$, and the poor performance of the local density approximation in describing the equilibrium volumes of $\mathrm{Ce}$ and the early actinides. A significant question is the importance of localization (i.e. f-electron correlation) in the rare-earths and actinides, particularly in $\mathrm{Ce}, \mathrm{Np}$, and $\mathrm{Pu}$, since the differences in structural properties between the transition metals and actinides have been taken as an indication of the breakdown of the itinerant electron description of these materials. Of further interest is the change in structural phase stability accompanying delocalization transitions in rare earths and actinides. The lack of an observed volume collapse accompanying these transitions in the post-Pu actindes is a serious discrepancy between experiment and theory. 


\section{Importance to LANL's Science and Technology Base and National R\&D Needs}

The work performed under this proposal has made significant advances in understanding such fundamental materials properties as structural phase stability, mechanical response, and the formation of magnetic moments. Our work on the structures and structural stability of actinides has contributed to understanding the equation of state and mechanical properties of these materials, benefiting the Laboratory's role in national defense. This work, together with the work on magnetic properties, has also contributed to the theory of electrons in actinides, a science base that is one of the Laboratory's particular strengths. Our contributions to the theory of the magnetic and mechanical properties of transition metals benefits Laboratory and national programs for the development of new structural materials and new materials for magnetic devices.

This work has relied heavily on LANL's capability to perform high-precision electronic structure calculations. All of the projects carried out have exercised and developed that capability, for example in the ability to go beyond the local density approximation. The capability of performing ab-initio calculations of materials properties has been and will continue to be an important part of LANL's science and technology base. This project supports Los Alamos core competencies in theory, modeling, and high-performance computing as well as nuclear weapons science and technology.

\section{Scientific Approach and Results to Date}

The work carried out under this project has resulted in 25 published manuscripts and several more being reviewed or prepared. Most of these are cited in the References.

One project has been the total energy calculation of magnetic anisotropy energies (MAE) in elemental $\mathrm{Fe}, \mathrm{Co}$, and $\mathrm{Ni}$ [3]. This work was the first successful description of this property in these elements. The results elucidated the role of orbital polarization in magnetocrysalline anisotropy and demonstrated conclusively the failure of the LDA in describing the easy axis of Ni. A by-product of this work was a new technique for Brillouin zone integrations [4]. The magnetic structure of the surface of $\mathrm{Fe}, \mathrm{Co}$, and $\mathrm{Ni}$, on $\mathrm{Cu}$ substrates has been studied. The Co surface was studied in conjunction with experimental work. A significant result was the finding of strongly enhanced orbital moments on the surface and demonstrating that this is a systematic phenomena $[5,6]$. Motivated by the apparent disagreement between experimental spin/orbital moments and Hund's third rule, calculations

were performed to demonstrate that parallel spin/orbital moments are induced by the fields used 
in the neutron experiments [7-9]. This work was extended to investigate field-induced magnetism in $U$ compounds [10]. Other work on magnetic properties carried out on this project included a study of the magnetization density in $\mathrm{CeFe}(2)$ [11], which found that the source of the experimentally observed anisotropy in that compound arises from orbital polarization, and the magnetic structure of Gd surfaces [12], which found ferromagnetic coupling to the bulk, in agreement with experiment and disagreement with previous calculations. Several other studies initiated under this project include studying the surface electronic and magnetic structure of alpha-Ce, the magnetic behavior of monolayers of Ru and $\mathrm{Rh}$ as well as $\mathrm{Ce}, \mathrm{U}$, and $\mathrm{Pu}$, on a $\mathrm{MgO}$ substrates, and calculations of the bulk magnetic anisotropy energy for US and $\mathrm{YCo}(5)$.

A significant result of our work on structural properties has been the demonstration that the crystal structure stabilities of both $d$ - and f-electron materials are driven by similar mechanisms. In particular, the unusual crystal structures of the light actinides may be understood as a consequence of normal itinerant electron bonding in a regime of narrow bandwidths [13-16]. This result is important in indicating that the structural properties of the actinides may be understood in the context of normal metallic bonding. Elasticity was studied in both actinide and transition metals. Elastic constants were calculated systematically for transition metal alloys with cubic symmetry. It was found that the tetragonal shear modulus is correlated with (cubic) structural energy differences and hence band-filling, and that the modulus of alloys can be predicted on this basis [17]. Similar work was done for the metallic 3-d elements in which it was demonstrated that crystal structure stabilities correlated with the filling of spin sub-bands and that the elastic constants correlated with this filling [18]. The elastic constants of La, Ce, and Th; cubic phases of $\mathrm{U}, \mathrm{Np}$, and Pu; and the compounds UC, US, and UTe were calculated to benchmark elastic constant calculations in the actinides to investigate the source of stability of cubic phases in the actinides and to study the unusual elastic properties observed experimentally in some uranium compounds. Agreement with experiment was good for La, Th, UC, and US, and the calculations for cubic phases supported temperature stabilized cubic phases in $\mathrm{U}, \mathrm{Np}$, and $\mathrm{Pu}$. Calculations for UTe failed to produce the negative Poisson ratio in that compound, a significant result in that the negative Poisson ratio observed experimentally has been suggested to be a result of correlated electron behavior. Our results reinforce this suggestion [19-21].

Electronic structure calculations for $\mathrm{Ce}$ and the light actinides have consistently given equilibrium volumes very different from experimental volumes. Although these calculations have generally been done for (hypothetical) fcc structures, the discrepancy has been suggested to be a fault of the itinerant electron description of the actinides. Motivated by the success of the recently formulated generalized gradient approximation (GGA) to density functional theory, 
the GGA was implemented in our electronic structure codes and used to re-calculate the equation of state of $\mathrm{Ce}$ and $\mathrm{Th}$ and the equilibrium properties of (hypothetical) fcc $\mathrm{U}, \mathrm{Np}$, and $\mathrm{Pu}$. The result was to bring the calculated volumes in agreement with experiment comparable to, for example, the transition metals [22-24]. Other puzzles in actinide theory that have been addressed in this project include the question of the increase in volume from $\mathrm{Np}$ to $\mathrm{Pu}$. Calculations are essentially complete for the equilibrium properties of $\mathrm{Np}$ and $\mathrm{Pu}$ in their experimentally observed crystal structures. The result is that the alpha-Pu structure has an anomolously high equilibrium volume, and hence the upturn from $\mathrm{Np}$ is a result of the open structure favored by $\mathrm{Pu}$. Work is continuing on another interesting puzzle in the actinides, namely the absence of experimentally observed volume collapses through delocalization transitions in actinides.

Structural stability has been studied in this project in a variety of other materials over a wide range of pressures [25-29]. This work has included a demonstration that the high pressure phase transition observed in $\mathrm{Sm}$ is a delocalization transition [25]. With few exceptions, it has been found in this work that the bcc structure is always favored at high pressures. We have demonstrated that, at least in the transition metals, this is a result of increasing hybridization between semi-core p-electrons and valence d-electrons [30].

\section{References}

[1] "Theoretical Studies of the High-Pressure Phases in Ce," J. M. Wills, Olle Eriksson, and A. M. Boring, Pysr. Rev. Lett. 67, 2215 (1991); "Crystal-structure stabilities and electronic structure for the light actinides Th, $\mathrm{Pa}$, and $\mathrm{U}$, " J. M. Wills and Olle Eriksson, Phys. Rev. B 45, 13879 (1992).

[2] "Prediction of Ferromagnetism and Metamagnetism in 4d Transition-Metal Overlayers on the (001) Surface of Ag ( $4 d=T c, R u, R h$, and Pd)", O. Eriksson, R. C. Albers, and A. M. Boring, Phys. Rev. Lett. 66, 1350 (1991).

[3] "Total Energy Calculation of the Magnetocrystalline Anisotropy Energy in the Ferromagnetic 3d Metals," J. Trygg, B. Johansson, O. Eriksson, and J. M. Wills, Phys. Rev. Lett., accepted.

[4] "Fermi Surface Averaging Technique for Calculating the Magnetocrystalline Anisotropy Energy," J. Trygg, J. M. Wills, B. Johansson, and O. Eriksson, Phys. Rev. B (submitted).

[5] "Inhancement of Orbital Magnetism at Surfaces: Co on $\mathrm{Cu}(100)$, , M. Tischer, O. Hjortstam, D. Arvanitis, J. Hunter Dunn, F. May, K. Baberschke, J. trygg, J. M. Wilss, B. Johansson, and O. Eriksson, Phys. Rev. Lett., accepted.

[6] "Calculated Spin and Orbital Moments in the 3d Metal Surfaces and 3d/Cu(001) Overlayers ( $3 \mathrm{~d}=\mathrm{Fe}, \mathrm{Co}$, and Ni)," O. Hjortstam, J. Trygg, J. M. Wills, B. Johansson, and O. Eriksson, to be submitted. 
[7] "Field-Induced Magnetism in f-Electron Systems: U, Pu, and Ce," Anders Hjelm, Joakim Trygg, Olle Eriksson, Borje Johansson, and John Wills, Phys. Rev. B. 50, 4332 (1994).

[8] "Field-Induced Magnetism in Actinide Systems," Anders Hjelm, Joakim Trygg, Olle Eriksson, Borje Johansson, and John M. Wills, J. Magn. Magn., 140-144, 1353 (1995).

[9] "Orbital Paramagnetism in Metallic Systems with Large Angular Momenta," A. Hjelm, J. Trygg, O. Eriksson, B. Johansson, and J. M. Wills, Modern Physics Letters A (invited).

[10] "Field Induced Magnetism in Uranium Compounds: UGe(3) and URh(3)," J. Trygg, J. M. Wills, B. Johansson, and O. Eriksson, Phys. Rev. B 50, 9226 (1994).

[11] "First Principles Study of the Magnetisation Density in CeFe(2),"J. Trygg, J. M. Wills, B. Johansson, and Olle Eriksson, Phys. Rev. B 50, 4200 (1994).

[12] "Bulk and Surface Magnetism and Interplanar Spacings in Gd from First-Principles Calculations," O. Eriksson, R. Ahuja, A. Ormeci, J. Trygg, O. Hjortstam, P. Soderlind, and J. M. Wills, Phys. Rev. B (accepted).

[13] "A Unified Picture of the Crystal Structures of Metals," Per Soderlind, Olle Eriksson, Borje Johansson, J. M. Wills, and A. M. Boring, Nature 374, 524 (1995).

[14] "Crystallographic Phase Transitions in Actinide Metals as a Function of Pressure," O. Eriksson, J. M. Wills, P. Soderlind, J. Melsen, R. Ahuja, A. M. Boring, and B. Johansson, Journal of Alloys and Compounds (accepted).

[15] "Theoretical Studies of the Crystal Structure of Rare-Earths and Actinides at Zero Temperature," J. M. Wills and O. Eriksson, in High. Press Science and Technology 93, editors: Schmidt, Scaner, Samara, and Ross, (AIP Press, NY, 1993) p.175.

[16] "First Principles Studies of $\mathrm{f}$ Elements," O. Eriksson, P. Soderlind, J. M. Wills, and A. M. Boring, Physica B 190, 12 (1993).

[17] "Theory of Elastic Constants of Cubic Transition Metals and Alloys, "Per Soderlind, Olle Eriksson, J. M. Wills, and A. M. Boring, Phys. Rev. B 48, 5844 (1993).

[18] "Crystal Structures and Elastic Constants of the Magnetic 3d Metals," P. Soderlind, R. Ahuja, O. Eriksson, J. M. Wills, Phys. Rev. B. 50, 5918 (1994).

[19] "Elastic Constants of Cubic f-Electron Elements: Theory," Per Soderlind, Olle Eriksson, J. M. Wills, and A. M. Boring, Phys. Rev. B, 48, 9306 (1993).

[20] "Elastic Constants of d Transition Elements and d Transition Alloys," P. Sodelrind, J. M. Wills, and O. Eriksson, in International Conference on the Physics of Transition Metals, editors P. M. Oppeneer and J.Kubler (World Scientific, Singapore, 1993).

[21] "Calculation of Elastic Constants in UC, US, and UTe, "J. Trygg, J. M. Wills, M. S. S. Brooks, B. Johansson, and O. Eriksson, Phys. Rev. B 52, 2492 (1995). 
[22] "Electronic Properties of f Electron Elements using the Generalised Gradient Approximation," P. Soderlind, O. Eriksson, B. Johansson, and J. M. Wills, Phys. Rev. B 50, 7291 (1994).

[23] "Density Functional Calculations on Cerium," P. Soderlind, O. Eriksson, B. Johansson, and J. M. Wills, Phys. Rev. B (accepted).

[24] "Theoretical Investigation of High Pressure Crystal Structures of alpha-Ce and Th," P. Soderlind, O. Eriksson, B. Johansson, and J. M. Wills, Phys. Rev. B, accepted.

[25] "Local-Moment Collapse in Compressed Samarium Metal," Per Soderlind, Olle Eriksson, J. M. Wills, and B. Johansson, Phys. Ref. B 48, 9212 (1993).

[26] "Crystal Structures of Ti, Zr, and Hf under Compression: Theory," R. Ahuja, J. M. Wills, B. Johansson, and Olle Eriksson, Phys. Rev. B 48, 16269 (1993).

[27] "Prediction of a bcc Structure in Compressed Yttrium," Joost Melsen, J. M. Wills, B. Johansson, and Olle Eriksson, Phys. Rev. B 48, 15574 (1993).

[28] "Theoretical Predictions of Structural Phase Transitions in Cr, Mo, and W," P. Soderlind, R. Ahuja, O. Eriksson, B. Johansson, and J. M. Wills, Phys. Rev. B 49, 9365 (1994).

[29] "Giant Surface Relaxation for Be (10-10)," O. Hjortstam, J. Trygg, J. M. Wills, B. Johansson, and O. Eriksson, Phys. Rev. Lett. (submitted).

[30] . "Influence of the Pseudocore p-Valence Band d-Hybridization on the Crystal Structure Phase Stabilities of Transition Metals under Extreme Pressures," R. Ahuja, P. Soderlind, J. Trygg, J. Melsen, J. M. Wills, B. Johansson, and O. Eriksson, Phys. Rev. B 50, 14690 (1994).

\title{
DISCLAIMER
}

\begin{abstract}
This report was prepared as an account of work sponsored by an agency of the United States Government. Neither the United States Government nor any agency thereof, nor any of their employees, makes any warranty, express or implied, or assumes any legal liability or responsibility for the accuracy, completeness, or usefulness of any information, apparatus, product, or process disclosed, or represents that its use would not infringe privately owned rights. Reference herein to any specific commercial product, process, or service by trade name, trademark, manufacturer, or otherwise does not necessarily constitute or imply its endorsement, recommendation, or favoring by the United States Government or any agency thereof. The views and opinions of authors expressed herein do not necessarily state or reflect those of the United States Government or any agency thereof.
\end{abstract}

